\title{
Transformations preserving norms of means of positive operators and nonnegative func- tions
}

\author{
LAJOS MOLNÁR AND PATRÍCIA SZOKOL
}

\begin{abstract}
Motivated by recent investigations on norm-additive and spectrally multiplicative maps on various spaces of functions, in this paper we determine all bijective transformations between the positive cones of standard operator algebras over a Hilbert space which preserve a given symmetric norm of a given mean of elements. A result of similar spirit is also presented concerning transformations between cones of nonnegative elements of certain algebras of continuous functions.
\end{abstract}

Mathematics Subject Classification (2010). Primary: 47B49. Secondary: 47A64, 26E60.

Keywords. Preservers, operator means, means of functions, symmetric norms.

\section{Introduction and statement of the results}

So-called norm-additive transformations and other closely related maps on function algebras have recently been studied by several authors, see, e.g., $[5,6,7,20,24]$. A map $\phi$ from an additive subsemigroup $S$ of a normed linear space to an additive subsemigroup $S^{\prime}$ of another normed linear space is called norm-additive if it satisfies

$$
\|\phi(x)+\phi(y)\|=\|x+y\|, \quad x, y \in S .
$$

The main point of investigating such transformations has been to find simple nonlinear characterizations of composition operators and algebra isomorphisms between function algebras. It is a trivial observation that dividing the sides of the equality in (1) by 2 , the so-obtained condition can be formulated

The authors were supported by the "Lendület" Program (LP2012-46/2012) of the Hungarian Academy of Sciences. The research was partially supported also by the European Union and the European Social Fund through project Supercomputer, the national virtual lab (grant no.: TAMOP-4.2.2.C-11/1/KONV-2012-0010). 
saying that $\phi$ preserves the norm of the arithmetic mean of elements. The study of norm-additive maps was preceded by that of so-called spectrally multiplicative maps. In the particular context of function algebras $C(X), C(Y)$ of all continuous complex valued functions on compact Hausdorff spaces $X, Y$, a map $\phi: C(X) \rightarrow C(Y)$ is called spectrally multiplicative if it satisfies

$$
\sigma(\phi(x) \phi(y))=\sigma(x y), \quad x, y \in C(X) .
$$

Here, $\sigma$ denotes the spectrum, which in the case of those function algebras equals the range of functions. The study of these maps was initiated in [13] by the first author and that paper influenced a number of subsequent works (according to Google Scholar, there are presently 87 citations to [13]). For very recent results see $[4,8,9,11,23]$, for a survey of the topic we refer to [6]. Observe that assuming $\phi$ sends nonnegative functions to nonnegative functions, taking square root in (2) and then taking sup-norm, we easily arrive at the equality $\|\sqrt{\phi(x) \phi(y)}\|=\|\sqrt{x y}\|$ for all nonnegative $x, y \in C(X)$. That means that $\phi$ preserves the norm of the geometric mean of nonnegative functions.

These really simple observations however put the original problems of norm-additive and spectrally multiplicative maps into another, much wider perspective. Recall that there are general theories of means of nonnegative numbers and positive operators and this brings the motivation to extend the investigations of norm-additive maps and spectrally multiplicative maps in that direction. In this paper we study transformations on structures of Hilbert space operators and scalar valued functions which preserve certain norms of general means of their positive or nonnegative elements and show how closely they are related to the isomorphisms of the underlying operator algebras and function algebras.

We first present our results concerning transformations on structures of positive operators that preserve a certain norm of a general mean of its elements. We emphasize that operator means play very important roles in several parts of mathematics with a rapidly increasing number of applications scattering from engineering sciences to quantum physics. In the papers $[15,16,17]$ the first author described the automorphisms of the set of all positive operators corresponding to means as algebraic operations. The present investigations are of different nature, here we study transformations which preserve certain geometrical numerical quantities attached to means, namely their certain norms.

Before presenting our results we fix the notation and collect the necessary information relating to means of positive operators. Let $H$ be a complex Hilbert space and denote by $B(H)$ the algebra of all bounded linear operators on $H$. We say that $A \in B(H)$ is positive and write $0 \leq A$ if $0 \leq\langle A x, x\rangle$ holds for every $x \in H$. We denote by $B(H)_{+}$the set of all positive elements of $B(H)$, this is called the positive cone of $B(H)$. Observe that here we use the concept of positivity in its usual operator theoretical sense which differs from its use in matrix theory where they would term it positive semidefinitness. 
For self-adjoint operators $A, B \in B(H)$ we write $A \leq B$ iff $B-A$ is positive. This gives us an order (more precisely a partial order) on the space of self-adjoint operators what we call the natural order. By a standard operator algebra over the Hilbert space $H$ we mean a subalgebra $\mathcal{A}$ of $B(H)$ which contains all finite rank operators in $B(H)$ and use the notation $\mathcal{A}_{+}$for the cone of all positive operators in $\mathcal{A}$.

The fundamentals of the famous Kubo-Ando theory of means of positive operators what we use in this paper are the following. As in the seminal paper [10], a binary operation $\sigma$ on $B(H)_{+}$is called a connection if it satisfies the following conditions. For any operators $A, B, C, D \in B(H)_{+}$and sequences $\left(A_{n}\right),\left(B_{n}\right)$ in $B(H)_{+}$we have

(O1) if $A \leq C$ and $B \leq D$ then $A \sigma B \leq C \sigma D$;

(O2) $C(A \sigma B) C \leq(C A C) \sigma(C B C)$;

(O3) if $A_{n} \downarrow A$ and $B_{n} \downarrow B$ then $A_{n} \sigma B_{n} \downarrow A \sigma B$,

where the arrow $\downarrow$ refers to monotone decreasing convergence in the strong operator topology. A connection $\sigma$ is called a mean if it is normalized in the sense that for the identity operator $I$ on $H$ we have $I \sigma I=I$. Clearly, for any connection (or mean) $\sigma$ on $B(H)_{+}$, its so-called transpose $\sigma^{t}$ defined by

$$
A \sigma^{t} B=B \sigma A, \quad A, B \in B(H)_{+}
$$

is a connection (or mean) again. The most simple means are the weighted arithmetic means (which are just the fixed convex combinations); $A \sigma B=$ $\lambda A+(1-\lambda) B$ with some given $\lambda \in[0,1]$.

One of the most important results in the Kubo-Ando theory says that there is an affine order-isomorphism between the class of all connections $\sigma$ on $B(H)_{+}$and the class of all nonnegative scalar valued operator monotone functions $f$ on $] 0, \infty[$, see Theorem 3.2 in [10]. In fact, as seen in the proof of that theorem, if $\sigma$ is a connection then the operator monotone function $f$ associated with it is defined by the formula $f(t)=I \sigma(t I), t>0$. Conversely, if $f$ is a nonnegative operator monotone function on $] 0, \infty[$ then the connection with which it is associated satisfies

$$
A \sigma B=A^{1 / 2} f\left(A^{-1 / 2} B A^{-1 / 2}\right) A^{1 / 2}
$$

for all invertible elements $A, B$ of $B(H)_{+}$. We remark that the property (O3) implies that the above formula extends also to the case where $A \in B(H)_{+}$ is invertible but $B \in B(H)_{+}$is arbitrary (to be correct, in that case the quantity $f(0)$ should be defined; we set $f(0)=\lim _{t \rightarrow 0} f(t)$ ). The case where $f(t)=\sqrt{t}, t>0$ is especially important. The corresponding mean is called the geometric mean of positive operators which has very many applications in different areas of science. By Corollary 4.2 in [10], if $\sigma$ is a connection with associated operator monotone function $f$ then $t \mapsto t f(1 / t), t>0$ is the operator monotone function on ] $0, \infty\left[\right.$ associated with the transpose $\sigma^{t}$ of $\sigma$.

By the famous Löwner theory of operator monotone functions it is wellknown that all such functions have a certain integral representation. In fact, 
the formula

$$
f(t)=\int_{[0, \infty]} \frac{t(1+s)}{t+s} d m(s), \quad t>0
$$

gives an affine order-isomorphism from the class of all positive Radon measures $m$ on the extended interval $[0, \infty]$ onto the set of all nonnegative scalar valued operator monotone functions $f$ on $] 0, \infty$ [, see Lemma 3.1 in [10]. We note that here $f(0):=\lim _{t \rightarrow 0} f(t)=m(\{0\})$ and $\lim _{t \rightarrow \infty} f(t) / t=m(\{\infty\})$. The integral representation (4) of operator monotone functions leads to an integral representation of connections. Namely, Theorem 3.4 in [10] tells us that for every connection $\sigma$ there exists a unique Radon measure $m$ on $[0, \infty]$ such that with $\alpha=m(\{0\})$ and $\beta=m(\{\infty\})$ we have

$$
A \sigma B=\alpha A+\beta B+\int_{] 0, \infty[} \frac{1+s}{s}\{s A: B\} d m(s)
$$

for any $A, B \in B(H)_{+}$. Here : denotes the so-called parallel sum of positive operators which is the half of the so-called harmonic mean; for invertible $A, B \in B(H)_{+}$we have $A: B=\left(A^{-1}+B^{-1}\right)^{-1}$.

In what follows we are going to describe the structure of all bijective transformations between the positive cones of standard operator algebras which preserve a given symmetric norm of a given Kubo-Ando mean of their elements. According to the behavior of the representing Radon measure of the mean we present our result in two theorems. In the first one we consider those means whose representing measure vanishes on $] 0, \infty[$. These are exactly the weighted arithmetic means $A \sigma B=\lambda A+(1-\lambda) B$ with given $\lambda \in[0,1]$. Observe that in the extreme cases where $\lambda=0$ or $\lambda=1$, the corresponding preserver transformations are exactly the bijective maps which preserve the norm of elements and clearly nothing more specific can be said about them or about their structure (we emphasize that we do not assume that the transformations under considerations have any linearity property in any sense). If $0<\lambda<1$, the situation is different as we see below.

Before presenting the first theorem we recall a few simple facts from operator theory. Let $1 \leq p<\infty$ be a given number and denote by $\operatorname{Tr}$ the usual trace functional. The symbol $C_{p}(H)$ stands for the set of all operators $A \in B(H)$ for which $\operatorname{Tr}|A|^{p}<\infty$. It is well-known that $C_{p}(H)$ is an ideal of $B(H)$ which is a Banach space under the $p$-norm defined by

$$
\|A\|_{p}=\left(\operatorname{Tr}|A|^{p}\right)^{1 / p}, \quad A \in C_{p}(H) .
$$

As for the case where $p$ is infinite, $C_{\infty}(H)$ denotes $B(H)$ equipped with the usual operator norm $\|$.$\| . The space C_{p}(H)$ is often called the Schatten - von Neumann $p$-class of operators.

Our first result relating to the particular case of weighted arithmetic means reads as follows.

Theorem 1. Let $H$ be a complex Hilbert space with $\operatorname{dim} H>1$ and $1<p \leq \infty$. Consider standard operator algebras $\mathcal{A}$ and $\mathcal{B}$ on $H$ which are contained in 
$C_{p}(H)$. Let $\phi: \mathcal{A}_{+} \rightarrow \mathcal{B}_{+}$be a bijective transformation such that for a given $0<\lambda<1$ we have that

$$
\|\lambda \phi(A)+(1-\lambda) \phi(B)\|_{p}=\|\lambda A+(1-\lambda) B\|_{p},
$$

holds for all $A, B \in \mathcal{A}_{+}$. Then there exists a unitary or antiunitary operator $U: H \rightarrow H$ such that

$$
\phi(A)=U A U^{*}, \quad A \in \mathcal{A}_{+} .
$$

One may immediately ask what happens in the case $p=1$. The answer is trivial, then the condition (6) means only that $\phi$ preserves the trace of the elements of $\mathcal{A}_{+}$which assumption has clearly no further consequence on the structure of $\phi$. Moreover, in relation with the above theorem we refer to the results in [19].

We next consider the case where the representing Radon measure $m$ of the given mean $\sigma$ vanishes either at 0 or at $\infty$ and present a result concerning a large class of norms. For this we need the notion of a symmetric norm on $B(H)$. We say that the norm $N$ on $B(H)$ is a symmetric norm if $N(A X B) \leq\|A\| N(X)\|B\|$ holds for all $A, B, X \in B(H)$. We point out that there are plenty of such norms, for example, among others, the so-called $(c, p)$ norms including the Ky Fan $k$-norms, see [3]. In the particular case where the underlying Hilbert space $H$ is finite-dimensional we have $C_{p}(H)=B(H)$ and the above mentioned $p$-norms are known to be symmetric norms, too.

In the next statement we consider bijective transformations between the positive cones of standard operator algebras $\mathcal{A}$ and $\mathcal{B}$ of $B(H)$ that preserve a given symmetric norm $N$ of a given mean $\sigma$ of all pairs of elements in the sense that $\phi$ satisfies

$$
N(\phi(A) \sigma \phi(B))=N(A \sigma B), \quad A, B \in \mathcal{A}_{+} .
$$

As mentioned above, here we deal with the case where the representing Radon measure $m$ of $\sigma$ vanishes either at 0 or at $\infty$. Observe that we may and do assume that $m(\{0\})=0$. Indeed, if $m(\{\infty\})=0$ and $\phi$ is a transformation from $\mathcal{A}_{+}$onto $\mathcal{B}_{+}$which satisfies (7) for all $A, B \in \mathcal{A}_{+}$, then $\phi$ satisfies (7) also for the transposed mean $\sigma^{t}$ and its representing measure vanishes at 0 (this follows from the general discussion above concerning operator means). We already know that $m(\{0\})=0$ is equivalent to $\lim _{t \rightarrow 0} f(t)=0$. But this condition itself is not sufficient to obtain any reasonable result. In fact, if $f$ is the identity then $A \sigma B=B$ for all $A, B \in B(H)_{+}$and (7) means only that $\phi$ preserves the $N$-norm of the elements which does not imply anything more concerning the structure of the transformation $\phi$. Fortunately, this is the only case what we need to rule out. The precise formulation of our second theorem reads as follows.

Theorem 2. Let $H$ be a complex Hilbert space with $\operatorname{dim} H>1$ and $N$ a symmetric norm on $B(H)$. Let $\mathcal{A}$ and $\mathcal{B}$ be standard operator algebras on $H$ and $\sigma$ a mean on $B(H)_{+}$such that the associated operator monotone function $f$ satisfies $\lim _{t \rightarrow 0} f(t)=0$ (which is equivalent to assuming that $I \sigma 0=0$ ) and $f$ is not the identity. If $\phi: \mathcal{A}_{+} \rightarrow \mathcal{B}_{+}$is a bijective transformation which 
satisfies (7) then there exists a unitary or antiunitary operator $U$ on $H$ such that

$$
\phi(A)=U A U^{*}, \quad A \in \mathcal{A}_{+} .
$$

After presenting our results concerning means on operator algebras, we now turn to the discussion of the problem concerning means on algebras of continuous functions. As for nonnegative functions (functions with nonnegative scalar values) the concept of means what we use is far more trivial than the notion of means of positive operators. In fact, it comes from the concept of means of nonnegative real numbers. We say that the function $M:[0, \infty[\times[0, \infty[\rightarrow[0, \infty[$ of two-variables is a mean on the nonnegative real numbers if the following hold

(N1) $\min \{a, b\} \leq M(a, b) \leq \max \{a, b\}, a, b \in[0, \infty[$;

(N2) for any $a, b, c, d \in[0, \infty[$, if $a \leq c$ and $b \leq d$ then $M(a, b) \leq M(c, d)$;

(N3) $M(\lambda a, \lambda b)=\lambda M(a, b), \lambda, a, b \in[0, \infty[$;

(N4) $M$ is continuous.

Just as in the case of operator means, to any such mean $M$ on nonnegative real numbers we can associate a real function $f:[0, \infty[\rightarrow[0, \infty[$ by the formula $f(t)=M(1, t), t \in[0, \infty[$. It is obvious that the function $f$ satisfies $f(1)=1$, it is continuous and monotone increasing, and the function $t \mapsto f(t) / t$ is monotone decreasing. Denote $\alpha=f(0)$ and $\beta=\lim _{t \rightarrow \infty} f(t) / t$. The mean $M$ can be recovered from $f$ in the following way

$$
M(a, b)= \begin{cases}\alpha a & \text { if } b=0, \\ \beta b & \text { if } a=0, \\ a f\left(\frac{b}{a}\right) & \text { if } a>0, b>0\end{cases}
$$

Conversely, let $f:[0, \infty[\rightarrow[0, \infty[$ be a continuous monotone increasing function such that $f(1)=1$ and assume that the function $t \mapsto f(t) / t, t>0$ is monotone decreasing. Then one can easily check that defining a two-variable function $M$ by the formula in (8), it satisfies all the conditions (N1)-(N4).

Let us remark that in the literature more general means on numbers are also studied in great details. Especially, the homogeneity property (N3) or the continuity (N4) may seem restrictive here but we assume them since for operator means both of them hold.

In what follows, for a locally compact Hausdorff space $X$ we denote by $C_{0}(X)$ the algebra of all complex valued continuous functions on $X$ that vanish at infinity. The norm $\|$.$\| what we consider on C_{0}(X)$ is the usual sup-norm. For any subalgebra $\mathcal{A}$ of $C_{0}(X)$ the symbol $\mathcal{A}_{+}$stands for the set of all functions in $\mathcal{A}$ whose values are nonnegative. Any mean $M$ on the nonnegative real numbers gives rise in an obvious way to a map that we call a mean on nonnegative functions and denote by the same symbol $M$. In fact, for all $x, y \in C_{0}(X)_{+}$we define $M(x, y)(t):=M(x(t), y(t)), t \in X$.

For our last result Theorem 3 we need the following. Let $X$ be a first countable locally compact Hausdorff space. It is known that for any point $t \in X$ there exists a function $p: X \rightarrow[0,1]$ with the following properties

(P) $p \in C_{0}(X)_{+}$has compact support, $p(t)=1$ and $p(s)<1$ if $s \neq t$. 
In this paper we call such a function $p$ a peaking function that peaks at $t$. (We mention that this is not the usual definition of peak functions what is commonly used in the theory of function algebras but for our purposes it is what we need.) To see that such a function exists for any $t \in X$, observe that by the first countability of $X$ there is a sequence $\left(U_{n}\right)$ of neighborhoods of $t$ which forms a base of neighborhoods of $t$. By a version of Urysohn's lemma, for any $n \in \mathbb{N}$ there exists a continuous function $p_{n}: X \rightarrow[0,1]$ with compact support in $U_{n}$ such that $p_{n}(t)=1$. Now the continuous function $p$ defined by $p=\sum_{n=1}^{\infty} \frac{1}{2^{n}} p_{n}$ has range in $[0,1]$, takes the value 1 exactly at $t$ and has compact support. So, $p$ is a peak function peaking at $t$. Furthermore, given any neighborhood $U$ of $t$ one can construct a continuous function $q$ with values in $[0,1]$ such that $q(t)=1$ and $\operatorname{supp} q \subset U$. Clearly, the product $p q$ is a peak function again peaking at $t$ and having compact support in the given neighborhood $U$.

Our theorem on the structure of transformations on nonnegative functions which preserve the norm of a given mean reads as follows. We remark that the condition that the peak functions all belong to the algebras what we consider in the next theorem is similar in spirit to the condition in our previous results concerning operator algebras which requires the inclusion of all finite rank operators.

Theorem 3. Let $X, Y$ be first countable locally compact Hausdorff spaces and suppose that $\mathcal{A} \subset C_{0}(X), \mathcal{B} \subset C_{0}(Y)$ are subalgebras which contain all peak functions on $X$, respectively on $Y$. Let $M$ be a mean on the nonnegative real numbers and let $f:[0, \infty[\rightarrow[0, \infty[$ be the real function associated with $M$. Assume $\phi: \mathcal{A}_{+} \rightarrow \mathcal{B}_{+}$is a bijective map with the property

$$
\|M(\phi(x), \phi(y))\|=\|M(x, y)\|, \quad x, y \in \mathcal{A}_{+} .
$$

In either of the two cases where

(i) $f(0)=0$ and the function $t \mapsto f(t) / t$ is strictly decreasing;

(ii) $\lim _{t \rightarrow \infty} f(t)=\infty, f$ is strictly increasing and $t \mapsto f(t) / t$ is strictly decreasing

there exists a homeomorphism $\varphi: Y \rightarrow X$ such that $\phi$ is of the form

$$
\phi(x)(t)=x(\varphi(t)), \quad t \in Y, x \in \mathcal{A}_{+} .
$$

For algebras of functions appearing in the statement above, one can get examples by considering any subalgebras of $C_{0}(X)$ which contain the subalgebra of all elements with compact support. Observe that this algebra includes the algebra generated by the collection of all peaking functions but usually different from that (it is so even in the simplest case $X=\mathbb{R}$ ). Therefore, there are many more examples.

We point out that the conditions that the function $t \mapsto f(t) / t$ is strictly decreasing, respectively that $f$ is strictly increasing are equivalent to that the mean $M$ to which $f$ is associated is strictly increasing in its first, respectively in its second variable. Hence the geometric mean provides a trivial example for (i). Also remark that (ii) above covers the case where $M$ is a weighted 
arithmetic mean, i.e., $M(a, b)=\lambda a+(1-\lambda) b, a, b \in[0, \infty[$ with some fixed $0<\lambda<1$.

The above results tell that the transformations under considerations all have particular forms. In the case of operator structures they are maps of the form $A \longmapsto U A U^{*}$ with some unitary or antiunitary operator $U$ while in the case of structures of scalar valued continuous functions they are of the form $x \longmapsto x \circ \varphi$ with a homeomorphism $\varphi$ between the underlying topological spaces. Let us recall that the algebra *-automorphisms of the operator algebra $B(H)$ are exactly the maps of the form $A \longmapsto U A U^{*}$ with some unitary operator $U$ on $H$ while its algebra *-antiautomorphisms are exactly the maps of the form $A \longmapsto U A^{*} U^{*}$ with some antiunitary operator $U$ on $H$. As for function algebras, it is well-known that the algebra isomorphisms between $C_{0}(X)$ and $C_{0}(Y)$ are exactly the maps of the form $x \longmapsto x \circ \varphi$ with some homeomorphism $\varphi: Y \rightarrow X$. Hence, it follows from our results that under certain conditions the bijective transformations that preserve a given norm of a given mean of elements in certain structures of positive Hilbert space operators or of nonnegative scalar valued continuous functions necessarily extend to algebra ${ }^{*}$-isomorphisms or algebra ${ }^{*}$-antiisomorphisms.

Finally, to conclude the introduction we remark that the converse statements in all presented results above are valid, too. We mean that transformations of the forms that appear in the conclusions of our theorems do have the considered preserver properties. Therefore, those results can be viewed as certain characterizations of algebra ${ }^{*}$-isomorphisms and ${ }^{*}$-antiisomorphisms of operator algebras and function algebras.

\section{Proofs}

In this section we present the proofs of our results. As for Theorem 1 we need the following characterization of the usual order $\leq$ between positive operators. We denote by $P_{1}(H)$ the set of all rank-one projections on the Hilbert space $H$. Clearly, every element $Q \in P_{1}(H)$ is of the form $Q=x \otimes x$ with some unit vector $x \in H$, where this latter operator is defined by $(x \otimes x) z=\langle z, x\rangle x$, $z \in H$.

Lemma. Let $1<p \leq \infty$ be a given number and pick positive operators $A, B \in$ $C_{p}(H)$. We have $A \leq B$ if and only if $\|A+t Q\|_{p} \leq\|B+t Q\|_{p}$ holds for any nonnegative real number $t$ and for every $Q \in P_{1}(H)$.

Proof. Assume first that $1<p<\infty$. Then the necessity part of the statement is clear. Indeed, it follows from the monotonicity of $p$-norms which is a wellknown fact. (For a statement of similar spirit concerning general symmetric norms on $C^{*}$-algebras that we shall use later, see Lemma 12 in [18].) To verify the sufficiency part we first assert that

$$
\lim _{t \rightarrow \infty}\left(\|A+t Q\|_{p}-t\right)=\operatorname{Tr} A Q .
$$


holds for any $A \in C_{p}(H)$ and $Q \in P_{1}(H)$. Indeed, since $\|t Q\|_{p}=t$, we obviously have

$$
\|A+t Q\|_{p}-t=\frac{\|(1 / t) A+Q\|_{p}-\|Q\|_{p}}{1 / t} .
$$

From Theorem 2.3 in [1] we know that the norm $\|\cdot\|_{p}$ is Fréchet differentiable at any point of $C_{p}(H)$. (We point out that this is not true for the operator norm.) In fact, from that theorem we also learn what the derivative of $\|\cdot\|_{p}$ is. Namely, computing that derivative at the point $Q$ in the direction of $A$ we have

$$
\lim _{t \rightarrow \infty}\left(\|A+t Q\|_{p}-t\right)=\lim _{t \rightarrow \infty} \frac{\|(1 / t) A+Q\|_{p}-\|Q\|_{p}}{1 / t}=\operatorname{Tr}\left(\frac{|Q|^{p-1} U^{*} A}{\|Q\|_{p}^{p-1}}\right),
$$

with $U$ being the partial isometry in the polar decomposition of $Q$. But clearly, $U=Q,|Q|^{p-1}=Q$ and $\|Q\|_{p}=1$ and hence we obtain (9).

Now, assume that $\|A+t Q\|_{p} \leq\|B+t Q\|_{p}$ holds for all nonnegative real numbers $t$ and for every rank-one projection $Q$ on $H$. Then applying (9) we deduce that

$$
\operatorname{Tr} A Q \leq \operatorname{Tr} B Q
$$

is true for every $Q \in P_{1}(H)$. But this trivially implies the inequality

$$
\langle A x, x\rangle \leq\langle B x, x\rangle
$$

for every unit vector $x \in H$ from which we infer $A \leq B$.

The case where $p=\infty$ needs to be handled in a different way. The "only if" part of the statement follows from the monotonicity of the operator norm. To see the "if" part we show that for any positive operator $A \in B(H)$ and rank-one projection $Q \in P_{1}(H)$ we again have

$$
\lim _{t \rightarrow \infty}(\|A+t Q\|-t)=\operatorname{Tr} A Q .
$$

To verify this, pick a unit vector $x \in H$ from the range of $Q$. We claim that for any positive real number $\epsilon$ the inequality

$$
A \leq(\langle A x, x\rangle+\epsilon) Q+t Q^{\perp}
$$

holds for large enough $t>0$. Indeed, take an arbitrary positive real number $\epsilon$. Setting $T=(\langle A x, x\rangle+\epsilon) Q+t Q^{\perp}$, we need to show that $T^{-1 / 2} A T^{-1 / 2} \leq I$ holds for large enough $t>0$. Obviously, we have

$$
\begin{gathered}
T^{-1 / 2} A T^{-1 / 2}=\frac{1}{\langle A x, x\rangle+\epsilon} Q A Q+\frac{1}{\sqrt{t} \sqrt{\langle A x, x\rangle+\epsilon}} Q A Q^{\perp} \\
+\frac{1}{\sqrt{t} \sqrt{\langle A x, x\rangle+\epsilon}} Q^{\perp} A Q+\frac{1}{t} Q^{\perp} A Q^{\perp} .
\end{gathered}
$$

The norm of $Q A Q=\langle A x, x\rangle Q$ equals $\langle A x, x\rangle$ and denoting the sum of the last three terms in the above displayed formula by $S_{t}$ we get that

$$
\left\|T^{-1 / 2} A T^{-1 / 2}\right\| \leq \frac{\langle A x, x\rangle}{\langle A x, x\rangle+\epsilon}+\left\|S_{t}\right\| .
$$


Clearly, as $t \rightarrow \infty$ we have that $S_{t}$ tends to 0 in the operator norm. Therefore, for large enough $t$ the right-hand side of the inequality above is less than 1 implying that $T^{-1 / 2} A T^{-1 / 2} \leq I$ also holds which is equivalent to the inequality (11). From that we infer that

$$
\|A+t Q\| \leq\left\|(\langle A x, x\rangle+\epsilon+t) Q+t Q^{\perp}\right\|
$$

is valid for large enough $t \geq 0$. It is obvious that the quantity $\langle A x, x\rangle+t$ is less than or equal to the left-hand side of the inequality (12). Moreover, the right-hand side of (12) trivially equals $\max \{\langle A x, x\rangle+\epsilon+t, t\}=\langle A x, x\rangle+\epsilon+t$. Therefore, for large enough $t \geq 0$ we have

$$
\begin{gathered}
0 \leq\|A+t Q\|-\langle A x, x\rangle-t \\
\leq\langle A x, x\rangle+\epsilon+t-\langle A x, x\rangle-t=\epsilon .
\end{gathered}
$$

This implies

$$
\lim _{t \rightarrow \infty}(\|A+t Q\|-t)=\langle A x, x\rangle=\operatorname{Tr} A Q .
$$

Now, assuming that for positive operators $A, B \in B(H)$ we have $\| A+$ $t Q\|\leq\| B+t Q \|$ for all $t \geq 0$ and $Q \in P_{1}(H)$, it follows from (10) that for any unit vector $x \in H$ we get

$$
\begin{gathered}
\langle A x, x\rangle=\lim _{t \rightarrow \infty}(\|A+t x \otimes x\|-t) \\
\leq \lim _{t \rightarrow \infty}(\|B+t x \otimes x\|-t)=\langle B x, x\rangle .
\end{gathered}
$$

Therefore, we conclude that $A \leq B$ and the proof of the lemma is complete.

Observe that the lemma fails to be true in the case where $p=1$.

Having proven the above characterization of the order among positive operators we are in a position to present the proof of Theorem 1.

Proof of Theorem 1. We give the proof only for the case where $\lambda=1 / 2$, the general case can be handled in the same way, only very trivial modifications are needed. Furthermore, we assume $p=\infty$, in the case where $1<p<\infty$ the argument is just the same.

So, assume that $\phi: \mathcal{A}_{+} \rightarrow \mathcal{B}_{+}$is a bijective transformation satisfying

$$
\|\phi(A)+\phi(B)\|=\|A+B\|, \quad A, B \in \mathcal{A}_{+} .
$$

We easily show that $\phi$ preserves the usual order $\leq$. Indeed, if for $A, B \in \mathcal{A}_{+}$ we have $A \leq B$ then the inequality $\|A+C\| \leq\|B+C\|$ holds for every $C \in \mathcal{A}_{+}$and hence we have $\|\phi(A)+\phi(C)\| \leq\|\phi(B)+\phi(C)\|$ for every $C \in \mathcal{A}_{+}$. Since $\phi$ is surjective, as $C$ ranges over the set $\mathcal{A}_{+}$we obtain that $\phi(C)$ ranges over the set $\mathcal{B}_{+}$. Therefore, we get that

$$
\|\phi(A)+t P\| \leq\|\phi(B)+t P\|
$$

holds for every $t \geq 0$ and rank-one projection $P \in \mathcal{B}_{+}$. By the previous lemma it follows that $\phi(A) \leq \phi(B)$. The same argument works for $\phi^{-1}$, too. Therefore, $\phi$ is an order-isomorphism between $\mathcal{A}_{+}$and $\mathcal{B}_{+}$. In particular, we obtain that $\phi(0)=0$. We next show that $\phi$ maps the rank-one elements of $\mathcal{A}_{+}$onto the rank-one elements of $\mathcal{B}_{+}$. In fact, this follows from the following 
easy observation. A nonzero operator $A$ in $\mathcal{A}_{+}$is rank-one if and only if any two elements of the set $\left\{B \in \mathcal{A}_{+}: 0 \leq B \leq A\right\}$ are comparable with respect to the order $\leq$. Using this characterization we get that for any $A \in \mathcal{A}_{+}$, the operator $A$ is rank-one if and only if so is $\phi(A)$. Rank-one projections are rank-one positive operators with norm 1 . Plugging $A=B$ in (13) we obtain that $\phi$ preserves the norm. Consequently, we deduce that $\phi$ maps the set $P_{1}(H)$ of rank-one projections onto itself.

We next assert that $\phi(t Q)=t \phi(Q)$ holds for every nonnegative real number $t$ and rank-one projection $Q$ on $H$. Indeed, for example for $t \geq 1$ (in the case where $t<1$ one can argue in a similar way), using the order preserving property of $\phi$ we get that $\phi(Q) \leq \phi(t Q)$. Since $\phi(Q)$ and $\phi(t Q)$ are rank-one operators, hence it follows that $\phi(t Q)$ is a scalar multiple of $\phi(Q)$. On the other hand, $\phi$ preserves the norm of any operator in $\mathcal{A}_{+}$which then implies $\phi(t Q)=t \phi(Q)$. Therefore, for arbitrary rank-one projections $P, Q$ in $\mathcal{A}_{+}$and nonnegative real number $t$ we have

$$
\|P+t Q\|=\|\phi(P)+\phi(t Q)\|=\|\phi(P)+t \phi(Q)\| .
$$

By (10) we have that $\|P+t Q\|-t \rightarrow \operatorname{Tr} P Q,\|\phi(P)+t \phi(Q)\|-t \rightarrow$ $\operatorname{Tr} \phi(P) \phi(Q)$ as $t \rightarrow \infty$. Therefore, we obtain that $\phi$ has the property that

$$
\operatorname{Tr} \phi(P) \phi(Q)=\operatorname{Tr} P Q, \quad P, Q \in P_{1}(H) .
$$

The structure of all such transformations on $P_{1}(H)$ is well-known. In fact, by Wigner's famous theorem on quantum mechanical symmetry transformations (see, e.g., p. 12 in [14]) it follows that there exists either a unitary or an antiunitary operator $U$ on $H$ such that

$$
\phi(P)=U P U^{*}, \quad P \in P_{1}(H) .
$$

It is now easy to complete the proof showing that the above equality extends to the whole set $\mathcal{A}_{+}$, too. Indeed, let $A$ be an arbitrary element of $\mathcal{A}_{+}$and pick an arbitrary rank-one projection $P \in P_{1}(H)$. For any $t \geq 0$, by the already known properties of $\phi$ it follows that

$$
\left\|U^{*} \phi(A) U+t P\right\|=\left\|\phi(A)+t U P U^{*}\right\|=\|\phi(A)+t \phi(P)\|=\|A+t P\| .
$$

Subtracting the number $t$ from both sides of this equality and letting $t$ tend to infinity, we obtain that

$$
\operatorname{Tr} U^{*} \phi(A) U P=\operatorname{Tr} A P
$$

holds for all $P \in P_{1}(H)$. This easily implies that $U^{*} \phi(A) U=A$ and hence we infer that $\phi(A)=U A U^{*}$ is valid for every element $A \in \mathcal{A}_{+}$. The proof is complete.

We now turn to the proof of Theorem 2. Our argument is based on a characterization of the usual order $\leq$ expressed in terms of means and symmetric norms which is be given in Lemma 4. Before that we present the necessary preliminaries. First we compute the operator $A \sigma P$ for any mean $\sigma$ with representing measure vanishing at 0 , arbitrary positive operator $A \in B(H)_{+}$and rank-one projection $P$ on $H$. To do this, we can follow the 
argument given in the proof of Lemma 2.6 in the paper [17] of the first author. Let us introduce the concept of the strength $\lambda(A, P)$ of a positive operator $A \in B(H)_{+}$along a rank-one projection $P \in P_{1}(H)$, see [2]. This is defined by the formula

$$
\lambda(A, P)=\sup \{t \geq 0: t P \leq A\}
$$

and the function $P \longmapsto \lambda(A, P)$ on $P_{1}(H)$ is called the strength function of $A$. The first author proved in Lemma 2 in [16] that for any $A \in B(H)_{+}$and $P \in P_{1}(H)$, the parallel sum $A: P$ of $A$ and $P$ satisfies

$$
A: P=\frac{\lambda(A, P)}{\lambda(A, P)+1} P .
$$

Now, using the integral representation (5) of $\sigma$ we get that

$$
A \sigma P=\alpha A+\beta P+\int_{] 0, \infty[} \frac{1+s}{s}\{s A: P\} d m(s) .
$$

On the other hand, let $f$ be the operator monotone function on $] 0, \infty[$ which is associated with the mean $\sigma$. By the integral representation (4) of $f$ we have that

$$
f(t)=\int_{[0, \infty]} \frac{t(1+s)}{t+s} d m(s)=\alpha+t \beta+\int_{] 0, \infty[} \frac{t(1+s)}{t+s} d m(s)
$$

holds for any $t>0$. Using (15), (16) and this representation, we deduce that if $t=\lambda(A, P)$ is positive then

$$
\begin{gathered}
A \sigma P=\alpha A+\beta P+\int_{] 0, \infty[} \frac{1+s}{s} \frac{s t}{s t+1} d m(s) P \\
=\alpha A+\beta P+t\left(f\left(\frac{1}{t}\right)-\alpha-\frac{\beta}{t}\right) P=\alpha A+t f\left(\frac{1}{t}\right) P-\alpha t P .
\end{gathered}
$$

Since the quantity $\alpha=m(\{0\})$ is supposed to be 0 , it implies that $A \sigma P=$ $t f(1 / t) P$.

Define the function $g:] 0, \infty[\rightarrow[0, \infty[$ by $g(t)=t f(1 / t), t>0$ which is just the operator monotone function associated with the transpose of $\sigma$. We have just learned that $A \sigma P=g(\lambda(A, P)) P$ holds whenever $\lambda(A, P)$ is positive. Moreover, if $\lambda(A, P)=0$ then by (15) and (16) we have $A \sigma P=\beta P$. On the other hand,

$$
g(0):=\lim _{t \rightarrow 0} g(t)=\lim _{t \rightarrow 0} t f(1 / t)=\lim _{t \rightarrow \infty} f(t) / t=m(\{\infty\})=\beta
$$

also holds and therefore we obtain that

$$
A \sigma P=g(\lambda(A, P)) P
$$

is valid in all possible cases.

Now, we are in a position to prove the following characterization of the order $\leq$ among positive operators. 
Lemma 4. Let $N$ be a symmetric norm on $B(H)$ and $\sigma$ a mean on $B(H)_{+}$ such that the associated operator monotone function $f$ satisfies $\lim _{t \rightarrow 0} f(t)=$ 0 and $f$ is not the identity. For any $A, B \in B(H)_{+}$we have

$$
A \leq B \Longleftrightarrow N(A \sigma C) \leq N(B \sigma C) \text { for every } C \in \mathcal{A}_{+} .
$$

Proof. By Lemma 12 in [18] we know that any symmetric norm on any $C^{*}$ algebra is monotone on the positive cone. This together with the monotonicity property (O1) of operator means imply that if $A, B$ in $\mathcal{A}_{+}$and $A \leq B$ then it follows that $N(A \sigma C) \leq N(B \sigma C)$ holds for every $C \in \mathcal{A}_{+}$. Conversely, assume that this inequality holds true for every $C \in \mathcal{A}_{+}$. Substituting $C=P$ for an arbitrary rank-one projection $P$ on $H$, the formula (17) gives us that $g(\lambda(A, P)) \leq g(\lambda(B, P))$. Observe that $g$ is neither identically 1 nor equals the identity which follow from the definition of $g$ given above and from the assumed properties of $f$. Since $g$ is an operator monotone function on $] 0, \infty[$, by Lemma 5.1 in [10] we then infer that $g$ is strictly increasing. Therefore we get $\lambda(A, P) \leq \lambda(B, P)$. Since this inequality holds for all rank-one projections $P$ on $H$, hence by Theorem 1 in [2] we obtain that $A \leq B$.

We next present the proof of Theorem 2. Below we shall use the fact that $\left(U A U^{*}\right) \sigma\left(U B U^{*}\right)=U(A \sigma B) U^{*}$ holds for any $A, B \in B(H)_{+}$and unitary or antiunitary operator $U$ on $H$. This equality follows easily e.g. from the formula (3) and the continuity property (O3) of connections.

Proof of Theorem 2. By the characterization given in Lemma $4, \phi$ is an orderisomorphism from $\mathcal{A}_{+}$onto $\mathcal{B}_{+}$. In particular, we have $\phi(0)=0$. Next, just as in the proof of Theorem 1 we can see that for any $A \in \mathcal{A}_{+}$we have $A$ is of rank 1 if and only if $\phi(A)$ is of rank 1 . Every positive rank-one operator is a positive scalar multiple of a rank-one projection. The rank-one projections are all unitarily equivalent and hence (since every symmetric norm is easily seen to be unitarily invariant), they have the same $N$-norm. We may and do assume in the following that this common norm-value is 1 which means that on positive rank-one operators the symmetric norm $N$ equals the usual operator norm. We learn from Theorem 3.3 in [10] that every mean $\sigma$ satisfies $A \sigma A=A$ for every operator $A \in B(H)_{+}$which implies that $\phi$ preserves the $N$-norm of all elements of $\mathcal{A}_{+}$. It follows that $\phi$ maps the set of rank-one projections (i.e., the set of all rank-one elements of $N$-norm equal to 1 ) onto itself.

In the following we show that for any $A \in \mathcal{A}_{+}$we have $A$ is a projection if and only if so is $\phi(A)$. Let $A$ be an element of $\mathcal{A}_{+}$. By (17) and the preserver properties of $\phi$, for any rank-one projection $P$ in $\mathcal{A}_{+}$we have

$$
g(\lambda(\phi(A), \phi(P)))=N(\phi(A) \sigma \phi(P))=N(A \sigma P)=g(\lambda(A, P)) .
$$

Since $g$ is strictly increasing (see the proof of Lemma 4), we deduce that

$$
\lambda(\phi(A), \phi(P))=\lambda(A, P) .
$$

In particular, this means that the range of the strength function of $A$ equals the range of the strength function of $\phi(A)$. From Lemma 4 in [2] we learn 
that $A$ is a projection if and only if $\lambda(A, P) \in\{0,1\}$ holds for any rankone projection $P$ on $H$. Using this characterization we can infer that $A$ is a projection if and only if so is $\phi(A)$.

Next, we assert that $\phi(P)$ is a rank- $n$ projection if and only if $P$ is a rank- $n$ projection. Indeed, the projection $P \in \mathcal{A}_{+}$has rank $n$ if and only if the maximal length of chains $P_{1}, \ldots, P_{k}$ of projections in $\mathcal{A}_{+}$for which $0 \supsetneqq P_{1} \supsetneqq P_{2} \supsetneqq \ldots \supsetneqq P_{k}=P$ holds is exactly $n$. Since $\phi$ is an orderisomorphism, it then follows that $P$ is a rank- $n$ projection if and only if so is $\phi(P)$.

We now prove that $\phi$ preserves the rank of any (finite rank) operator in $\mathcal{A}_{+}$. Let $P \in \mathcal{A}_{+}$be a finite rank projection and $t \geq 1$ a real number. We claim that $\phi(t P)=t \phi(P)$. Clearly, the strength function of $t P$ has range $\{0, t\}$. By the already established properties of $\phi$, the same holds for the strength function of $\phi(t P)$. It then follows that $\phi(t P)$ is of the form $\phi(t P)=t Q$ with some projection $Q \in \mathcal{B}_{+}$. But we have $Q=\phi\left(P^{\prime}\right)$ for some projection $P^{\prime} \in \mathcal{A}_{+}$. Furthermore, for any projection $R \in \mathcal{A}_{+}$we argue

$$
R \leq P \Leftrightarrow R \leq t P \Leftrightarrow \phi(R) \leq t \phi\left(P^{\prime}\right) \Leftrightarrow \phi(R) \leq \phi\left(P^{\prime}\right) \Leftrightarrow R \leq P^{\prime}
$$

which apparently implies $P=P^{\prime}$ and thus proving our claim $\phi(t P)=t \phi(P)$. Now, for any rank- $n$ operator $A \in \mathcal{A}_{+}$we have a rank- $n$ projection $P$ and a number $t \geq 1$ such that $A \leq t P$. It follows that $\phi(A) \leq t \phi(P)$ from which we infer that the rank of $\phi(A)$ is at most $n$. Therefore, $\phi$ is rank nonincreasing. Since $\phi$ and $\phi^{-1}$ share the same preserver properties, we deduce that $\phi$ is in fact rank preserving.

We say that an operator acts on the closed subspace $H_{0}$ of $H$ if $H_{0}$ is an invariant subspace of the operator and it is zero on the orthogonal complement of $H_{0}$. Clearly, the finite rank operator $A \in \mathcal{A}_{+}$acts on the range of the finite rank projection $P$ if and only if $A \leq t P$ holds for some real number $t \geq 1$. But that operator inequality is equivalent to $\phi(A) \leq \phi(t P)=$ $t \phi(P)$. This implies that $A \in \mathcal{A}_{+}$acts on the range of a rank-n projection $P$ if and only if $\phi(A)$ acts on the range of the rank- $n$ projection $\phi(P)$.

From this point we can literally follow the argument presented in [12] on page 5907 from the second to the fourth paragraph and prove, by making use of a beautiful theorem of Rothaus, that the order-isomorphism $\phi$ is necessarily additive and positive homogeneous on the cone $F(H)_{+}$of positive elements of the ideal $F(H)$ of all finite rank operators in $B(H)$. Consider the restriction $\phi_{\mid F(H)_{+}}$of $\phi$ onto $F(H)_{+}$. We can extend this map in an obvious way first to a bijective real linear map on the space of all finite-rank self-adjoint operators in $B(H)$ (where we use the fact that every self-adjoint operator in $F(H)$ is the difference of two positive ones) and then further extend it to a bijective complex linear map $\phi_{0}$ on the whole space $F(H)$. Just as $\phi_{\mid F(H)_{+}}$, this map $\phi_{0}$ also sends projections to projections and hence, e.g. by the argument in Theorem A.4 in [14], it is a so-called Jordan *-automorphism of $F(H)$ (meaning that it is a linear transformation which satisfies $\phi_{0}\left(A^{2}\right)=\phi_{0}(A)^{2}$ and $\phi_{0}\left(A^{*}\right)=\phi_{0}(A)^{*}$ for every $\left.A \in F(H)\right)$. The structure of those transformations is well-known. For example, by Theorems A.7 and A.8 in [14], $\phi_{0}$ is 
necessarily either of the form $A \longmapsto U A U^{*}$ with a unitary operator $U$ or of the form $A \longmapsto U A^{*} U^{*}$ with an antiunitary operator $U$ on $H$. It follows that we have $\phi(A)=U A U^{*}$ for all finite rank elements $A$ of $\mathcal{A}_{+}$.

Recalling that any symmetric norm is unitary invariant, if $A \in \mathcal{A}_{+}$is arbitrary then for every rank-one projection $P$ on $H$ we can compute

$$
\begin{gathered}
g(\lambda(A, P))=N(g(\lambda(A, P)) P)=N(A \sigma P) \\
=N(\phi(A) \sigma \phi(P))=N\left(\phi(A) \sigma\left(U P U^{*}\right)\right)=N\left(U\left(\left(U^{*} \phi(A) U\right) \sigma P\right) U^{*}\right) \\
=N\left(\left(U^{*} \phi(A) U\right) \sigma P\right)=g\left(\lambda\left(U^{*} \phi(A) U, P\right)\right) .
\end{gathered}
$$

Therefore, we have $\lambda(A, P)=\lambda\left(U^{*} \phi(A) U, P\right)$ for all rank-one projections $P$ on $H$ and applying Theorem 1 in [2] we infer that $U^{*} \phi(A) U=A$. Consequently, for any $A \in \mathcal{A}_{+}$we have $\phi(A)=U A U^{*}$ and this completes the proof.

We have seen above that order automorphisms of the positive cone of the algebra of finite rank operators play an essential role in the proof of Theorem 2. In fact, above we have followed the approach to such maps which has originally been invented in the paper [12] of the first author. We point out that in [21] (also see [22]) Šemrl has significantly improved the results of [12] by making use of the very original and effective idea of employing adjacency preserving maps.

Now, we turn to the proof of Theorem 3. We shall need the following lemma which gives a characterization of the natural order $\leq$ between nonnegative functions in terms of the uniform norm of means. Here by the order $\leq$ between scalar valued functions we mean the pointwise order, i.e., for any real valued functions $x, y \in C_{0}(X)$ we have $x \leq y$ if and only if $x(t) \leq y(t)$ holds for all $t \in X$.

Lemma 5. Let $\mathcal{A}$ be as in Theorem 3. Let $M$ be a mean on the nonnegative real numbers and denote by $f:[0, \infty[\rightarrow[0, \infty[$ the real function associated with $M$. Assume that the function $t \mapsto f(t) / t, t>0$ is strictly decreasing and either $f(0)=0$ or $\lim _{t \rightarrow \infty} f(t)=\infty$ holds. Then for any $x, y \in \mathcal{A}_{+}$we have

$$
x \leq y \Longleftrightarrow\|M(x, z)\| \leq \| M(y, z)) \| \text { for every } z \in \mathcal{A}_{+} .
$$

Proof. Since $M$ is a mean, it is increasing in its variables and hence the necessity part of the lemma is obvious. For the sufficiency suppose that $\|M(x, z)\| \leq\|M(y, z)\|$ holds for every $z \in \mathcal{A}_{+}$. Let $t_{0}$ be any point of $X$ and choose an arbitrary real number $\epsilon>0$. By the continuity of $y$ there exists a neighborhood $U$ of $t_{0}$ such that $y(t) \leq y\left(t_{0}\right)+\epsilon, t \in U$. Choose a peaking function $z$ on $X$ with compact support in $U$ that peaks at $t_{0}$ (see the discussion preceding the formulation of Theorem 3). Then $z \in \mathcal{A}_{+}$.

Assume first that $f(0)=0$. For every $t \notin U$ we have

$$
M(y(t), z(t))=M(y(t), 0)=y(t) f(0)=0 \leq\left(y\left(t_{0}\right)+\epsilon\right) f\left(1 /\left(y\left(t_{0}\right)+\epsilon\right)\right),
$$

while for $t \in U$ we compute

$$
M(y(t), z(t)) \leq M\left(y\left(t_{0}\right)+\epsilon, 1\right)=\left(y\left(t_{0}\right)+\epsilon\right) f\left(1 /\left(y\left(t_{0}\right)+\epsilon\right)\right) .
$$


Therefore, if $x\left(t_{0}\right) \neq 0$ then using $z\left(t_{0}\right)=1$ we deduce

$$
\begin{gathered}
x\left(t_{0}\right) f\left(1 / x\left(t_{0}\right)\right)=M\left(x\left(t_{0}\right), z\left(t_{0}\right)\right) \leq\|M(x, z)\| \\
\leq\|M(y, z)\| \leq\left(y\left(t_{0}\right)+\epsilon\right) f\left(1 /\left(y\left(t_{0}\right)+\epsilon\right)\right)
\end{gathered}
$$

which, by the strict decreasing property of the function $t \mapsto f(t) / t$, implies that $x\left(t_{0}\right) \leq y\left(t_{0}\right)+\epsilon$. Clearly, this inequality holds true also in the case where $x\left(t_{0}\right)=0$. Letting $\epsilon$ tend to 0 , we infer $x\left(t_{0}\right) \leq y\left(t_{0}\right)$. Since $t_{0} \in X$ is arbitrary, it follows that $x \leq y$.

Assume now that $\lim _{t \rightarrow \infty} f(t)=\infty$. For any positive real number $r$ and $t \in U$, the inequality

$$
M(y(t), r z(t)) \leq M\left(y\left(t_{0}\right)+\epsilon, r\right)=\left(y\left(t_{0}\right)+\epsilon\right) f\left(r /\left(y\left(t_{0}\right)+\epsilon\right)\right)
$$

holds. By the boundedness of $y$ and the unboundedness of $f$ we can choose $r$ such that for all $t \notin U$ we also have

$M(y(t), r z(t))=M(y(t), 0) \leq \max \{y(t), 0\}=y(t) \leq\left(y\left(t_{0}\right)+\epsilon\right) f\left(r /\left(y\left(t_{0}\right)+\epsilon\right)\right)$.

Therefore, if $x\left(t_{0}\right) \neq 0$, we infer

$$
\begin{gathered}
x\left(t_{0}\right) f\left(r / x\left(t_{0}\right)\right)=M\left(x\left(t_{0}\right), r z\left(t_{0}\right)\right) \leq\|M(x, r z)\| \\
\leq\|M(y, r z)\| \leq\left(y\left(t_{0}\right)+\epsilon\right) f\left(r /\left(y\left(t_{0}\right)+\epsilon\right)\right)
\end{gathered}
$$

which implies just as above that $x\left(t_{0}\right) \leq y\left(t_{0}\right)+\epsilon$. One can complete the proof as in the former case.

We are now in a position to prove Theorem 3.

Proof of Theorem 3. First, from $\|M(\phi(x), \phi(x))\|=\|M(x, x)\|$ we deduce that $\|\phi(x)\|=\|x\|$ holds for any $x \in \mathcal{A}_{+}$, i.e., $\phi$ preserves the norm. From the previous lemma it follows that for any $x, y \in \mathcal{A}_{+}$we have

$$
x \leq y \Longleftrightarrow \phi(x) \leq \phi(y)
$$

hence $\phi$ is an order-isomorphism. In particular, we get $\phi(0)=0$.

We next show that for any $x, y \in \mathcal{A}_{+}$we have

$$
x y=0 \Longleftrightarrow \phi(x) \phi(y)=0 .
$$

In fact, using the order preserving property of $\phi$, this immediately follows from the following easy observation. For any $x, y \in \mathcal{A}_{+}$we have

$$
x y=0 \Longleftrightarrow\left(\forall z \in \mathcal{A}_{+}: \quad z \leq x, y \Rightarrow z=0\right) .
$$

The implication $\Longrightarrow$ is obvious. To see the converse, assume $x y \neq 0$. Clearly, we can assume that the norm of $x$ and $y$ both equal 1 . It is clear that $0 \neq$ $v=x y \in \mathcal{A}_{+}$and $v \leq x, y$. Therefore, we have the equivalence in the above displayed formula and then (18) follows.

Next we show that for any peaking function $x \in \mathcal{A}_{+}$which peaks at a point $t \in X$, the function $\phi(x)$ is also a peaking function that peaks at some point $\varphi(t) \in Y$. First observe that since $\phi$ preserves the norm, we have $\|\phi(x)\|=1$. We need to show that $\phi(x)$ attains its maximum value 1 at a unique point in $Y$. To see this, assume that $\phi(x)$ takes this value at two different points, say $t, s \in Y$. We can choose peaking functions $u, v \in \mathcal{B}_{+}$such 
that $u$ peaks at $t$ and $v$ peaks at $s, u v=0$ and $u+v=\max \{u, v\} \leq \phi(x)$. Since $\phi$ is an order-isomorphism, we deduce

$$
\phi^{-1}(u) \leq \phi^{-1}(u+v) \leq x \text { and } \phi^{-1}(v) \leq \phi^{-1}(u+v) \leq x .
$$

On the other hand, by (18), $\phi$ preserves zero product in both directions which implies that $\phi^{-1}(u) \phi^{-1}(v)=0$. Therefore, the functions $\phi^{-1}(u)$ and $\phi^{-1}(v)$ of norm 1 take the value 1 at two different points and then, by (19), the same must be true for $x$. Since this is a contradiction, we obtain that $\phi(x)$ is really a peaking function. As $\phi, \phi^{-1}$ share similar properties, we can infer that $\phi$ preserves peaking functions in both directions meaning that $x \in \mathcal{A}_{+}$ is a peaking function if and only if $\phi(x) \in \mathcal{B}_{+}$is a peaking function.

In the next step we show that the point $\varphi(t)$ does not depend on the particular choice of the peaking function $x \in \mathcal{A}_{+}$that peaks at the point $t$. For any $x \in \mathcal{A}_{+}$(or $x \in \mathcal{B}_{+}$) denote by $\mathcal{F}_{x}$ the set of all peaking functions $z$ in $\mathcal{A}_{+}$(or in $\mathcal{B}_{+}$) which are below $x$, i.e., for which $z \leq x$ holds. Since $\phi$ preserves the order and peaking functions in both directions, we clearly have $\phi\left(\mathcal{F}_{x}\right)=\mathcal{F}_{\phi(x)}, x \in \mathcal{A}_{+}$. It is easy to check that if $x, y \in \mathcal{A}_{+}$are peaking functions, $x$ peaks at $t \in X, y$ peaks at $s \in X$, then

$$
t \neq s \Longleftrightarrow \mathcal{F}_{x} \cap \mathcal{F}_{y}=\emptyset \text {. }
$$

Indeed, the implication $\Longrightarrow$ is trivial. As for the converse, if $t=s$ then $x y \in \mathcal{F}_{x} \cap \mathcal{F}_{y}$ verifying the above equivalence. It implies that for any pair $x, y$ of peaking functions in $\mathcal{A}_{+}$, they peak at the same point if and only if the same holds for the peaking functions $\phi(x), \phi(y)$ in $\mathcal{B}_{+}$. It follows that $\varphi(t)$ is a well-defined point in $Y$ and hence we have a map $\varphi: X \rightarrow Y$ associated to $\phi$. It is obvious that there is a map $\psi: Y \rightarrow X$ associated to $\phi^{-1}$, too, and we clearly have that $\psi \circ \varphi, \varphi \circ \psi$ are both identities. It follows that $\varphi, \psi$ are bijections.

In what follows we show that $\phi$ is of the desired form. Let $t_{0}$ be an arbitrary point in $X$. Pick $y \in \mathcal{A}_{+}$. Just as in the proof of Lemma 5 , there exists a neighborhood $U$ of $t_{0}$ such that $y(t) \leq y\left(t_{0}\right)+\epsilon, t \in U$. Let $z$ be the peaking function in $\mathcal{A}_{+}$which peaks at $t_{0}$ and has compact support in $U$ that we have chosen in that proof. It follows from the argument given there that

$$
\|M(y, z)\| \leq\left(y\left(t_{0}\right)+\epsilon\right) f\left(1 /\left(y\left(t_{0}\right)+\epsilon\right)\right)
$$

holds in the case where $f(0)=0$ and we have

$$
\|M(y, r z)\| \leq\left(y\left(t_{0}\right)+\epsilon\right) f\left(r /\left(y\left(t_{0}\right)+\epsilon\right)\right)
$$

for some positive real number $r$ in the case where $\lim _{t \rightarrow \infty} f(t)=\infty$. Since $\phi(z)$ is a peaking function which peaks at $\varphi\left(t_{0}\right)$, assuming $\phi(y)\left(\varphi\left(t_{0}\right)\right) \neq 0$, in the case (i) we compute

$$
\begin{aligned}
& \phi(y)\left(\varphi\left(t_{0}\right)\right) f\left(1 /\left(\phi(y)\left(\varphi\left(t_{0}\right)\right)\right)\right)=M\left(\phi(y)\left(\varphi\left(t_{0}\right)\right), \phi(z)\left(\varphi\left(t_{0}\right)\right)\right) \\
& \leq\|M(\phi(y), \phi(z))\|=\|M(y, z)\| \leq\left(y\left(t_{0}\right)+\epsilon\right) f\left(1 /\left(y\left(t_{0}\right)+\epsilon\right)\right) .
\end{aligned}
$$

In the case (ii) the situation is more complicated. So, assume (ii) holds. We claim that $\phi(r z)\left(\varphi\left(t_{0}\right)\right)=r$. To verify this, for any $x \in \mathcal{A}_{+}$denote by 
$E(x)$ the set of all points in $X$ at which $x$ attains its maximum, i.e., set $E(x)=\{t \in X \mid x(t)=\|x\|\}$. Using the strict monotonicity of $M$ in its both variables, one can easily check that for any $x, u \in \mathcal{A}_{+}$

$$
E(x) \cap E(u) \neq \emptyset \Longleftrightarrow\|M(x, u)\|=M(\|x\|,\|u\|) .
$$

This obviously implies that for any $x, u \in \mathcal{A}_{+}$we have

$$
E(x) \cap E(u) \neq \emptyset \Longleftrightarrow E(\phi(x)) \cap E(\phi(u)) \neq \emptyset .
$$

Next, we show that for all $x, u \in \mathcal{A}_{+}$

$$
E(x) \subset E(u) \Longleftrightarrow E(\phi(x)) \subset E(\phi(u)) .
$$

Clearly, by (21), this is proven if we show that

$$
E(x) \subset E(u) \Longleftrightarrow\left(\forall v \in \mathcal{A}_{+}: E(x) \cap E(v) \neq \emptyset \Rightarrow E(u) \cap E(v) \neq \emptyset\right) .
$$

The implication $\Longrightarrow$ is trivial. As for the converse, assume that there exists an element $t$ in $E(x)$ which does not belong to $E(u)$. Clearly, $E(u)$ is a closed set. Select a peaking function $v$ with compact support in $E(u)^{c}$ which peaks at $t$. It follows that $v \in \mathcal{A}_{+}$and we obtain that $t \in E(x) \cap E(v)$ but the intersection $E(u) \cap E(v)$ is empty. By this contradiction we obtain the equivalence (22). It is obvious that we then also have that

$$
E(x)=E(u) \Longleftrightarrow E(\phi(x))=E(\phi(u))
$$

holds for all $x, u \in \mathcal{A}_{+}$.

After this, considering the peaking function $z \in \mathcal{A}_{+}$again which peaks at $t_{0}$ and any positive real number $r$, since $E(z)=E(r z)$, it follows that $\left.\left\{\varphi\left(t_{0}\right)\right)\right\}=E(\phi(z))=E(\phi(r z))$. We know that $\phi$ preserves the norm of functions, hence we deduce $\phi(r z)\left(\varphi\left(t_{0}\right)\right)=r$.

Now, assuming $\phi(y)\left(\varphi\left(t_{0}\right)\right) \neq 0$ we compute

$$
\begin{aligned}
& \phi(y)\left(\varphi\left(t_{0}\right)\right) f\left(r /\left(\phi(y)\left(\varphi\left(t_{0}\right)\right)\right)\right)=M\left(\phi(y)\left(\varphi\left(t_{0}\right)\right), \phi(r z)\left(\varphi\left(t_{0}\right)\right)\right) \\
\leq & \|M(\phi(y), \phi(r z))\|=\|M(y, r z)\| \leq\left(y\left(t_{0}\right)+\epsilon\right) f\left(r /\left(y\left(t_{0}\right)+\epsilon\right)\right)
\end{aligned}
$$

By the strict monotonicity of the function $t \mapsto f(t) / t$, from both equations (20), (23) we infer that

$$
\phi(y)\left(\varphi\left(t_{0}\right)\right) \leq y\left(t_{0}\right)+\epsilon
$$

holds true and hence letting $\epsilon$ converge to 0 we deduce $\phi(y)\left(\varphi\left(t_{0}\right)\right) \leq y\left(t_{0}\right)$. Since $t_{0} \in X$ is arbitrary we obtain $\phi(y) \leq y \circ \varphi^{-1}$. Clearly, similar inequality holds for $\phi^{-1}$ and its associated map $\psi=\varphi^{-1}$, too, i.e., we have $y=\phi^{-1}(\phi(y)) \leq \phi(y) \circ \varphi$. This implies that $\phi(y)=y \circ \varphi^{-1}$ is valid for every $y \in \mathcal{A}_{+}$.

It remains to prove that $\varphi$ is a homeomorphism which follows from a well-known argument. First observe that by the similar properties of $\phi, \phi^{-1}$ and those of the associated maps $\varphi, \psi$ we need only to show that $\varphi$ is continuous. Let $t \in X,\left(t_{n}\right)$ be a sequence in $X$ which converges to $t$ and suppose that $\varphi\left(t_{n}\right)$ does not converge to $\varphi(t)$. Then there exists a neighborhood $V \subset Y$ of $\varphi(t)$ such that $\varphi\left(t_{n}\right) \notin V$ for infinitely many indices. Let $x^{\prime} \in \mathcal{B}_{+}$, $x^{\prime}: Y \rightarrow[0,1]$ be a function such that $x^{\prime}(\varphi(t))=1$ and $\operatorname{supp}\left(x^{\prime}\right) \subset V$ and 
pick $x \in \mathcal{A}_{+}$such that $\phi(x)=x^{\prime}$. We have that $x\left(t_{n}\right)=x^{\prime}\left(\varphi\left(t_{n}\right)\right)=0$ for infinitely many indices which contradicts $x\left(t_{n}\right) \rightarrow x(t)=x^{\prime}(\varphi(t))=1$. This completes the proof of the theorem.

We conclude the paper with some remarks. We recall that in the first two theorems we assumed that $\operatorname{dim} H>1$. If this dimension is 1 then we in fact have numbers instead of operators and then our third theorem trivially applies. Much more importantly, we mention that not all possible means are considered in Theorems 1,2,3. For example, as for our results relating to operator means, we do not know what happens in the case where the representing measure $m$ of the mean $\sigma$ has the property that the values $m(\{0\}), m(\{\infty\}) m(] 0, \infty[))$ are all positive. However, the most important cases represented by the arithmetic mean, geometric mean, harmonic mean (double of the parallel sum) and many others are covered. There is room for further considerations in other respects, too. It would be good to know if Theorem 1 is valid also for some general classes of symmetric norms on $B(H)$ (not including the trace norm, of course). As for Theorem 3, it seems to be a natural question to explore if the result is true for the algebras $\mathcal{A}=C_{0}(X)$, $\mathcal{B}=C_{0}(Y)$ when the spaces $X, Y$ are not first countable, as well as to consider means of functions originating from more general means on nonnegative real numbers (e.g., nonhomogeneous means).

\section{Acknowledgements}

The authors express their thanks to the referee for the careful reading of the manuscript and for pointing out some flaws in the presentation.

\section{References}

1. T.J. Abatzoglou, Norm derivatives on spaces of operators, Math. Ann. 239 (1979), 129-135.

2. P. Busch and S.P. Gudder, Effects as functions on projective Hilbert spaces, Lett. Math. Phys., 47 (1999), 329-337.

3. J.T. Chan, C.K. Li and C.C.N. Tu, A class of unitarily invariant norms on $B(H)$, Proc. Amer. Math. Soc. 129 (2001), 1065-1076.

4. O. Hatori, A. Jiménez-Vargas and M. Villegas-Vallecillos, Maps which preserve norms of non-symmetrical quotients between groups of exponentials of Lipschitz functions, J. Math. Anal. Appl. 415 (2014), 825-845.

5. O. Hatori, G. Hirasawa and T. Miura, Additively spectral-radius preserving surjections between unital semisimple commutative Banach algebras, Cent. Eur. J. Math. 8 (2010), 597-601.

6. O. Hatori, S. Lambert, A. Luttman, T. Miura, T. Tonev, and R. Yates Spectral preservers in commutative Banach algebras, in Function Spaces in Modern Analysis, K. Jarosz, Edt. Contemporary Mathematics vol. 547, American Mathematical Society, 2011, pp. 103-124. 
7. M. Hosseini and J.J. Font, Norm-additive in modulus maps between function algebras, Banach J. Math. Anal. 8 (2014), 79-92.

8. M. Hosseini and F. Sady, Maps between Banach function algebras satisfying certain norm conditions, Cent. Eur. J. Math. 11 (2013), 1020-1033.

9. A. Jiménez-Vargas, K. Lee, A. Luttman and M. Villegas-Vallecillos, Generalized weak peripheral multiplicativity in algebras of Lipschitz functions, Cent. Eur. J. Math. 11 (2013), 1197-1211.

10. F. Kubo and T. Ando, Means of positive linear operators, Math. Ann., 246 (1980), 205-224.

11. K. Lee, Characterizations of peripherally multiplicative mappings between real function algebras, Publ. Math. Debrecen 84 (2014), 379-397.

12. L. Molnár, Order-automorphisms of the set of bounded observables, J. Math. Phys. 42 (2001), 5904-5909.

13. L. Molnár, Some characterizations of the automorphisms of $B(H)$ and $C(X)$, Proc. Amer. Math. Soc. 130 (2002), 111-120.

14. L. Molnár, Selected Preserver Problems on Algebraic Structures of Linear Operators and on Function Spaces, Lecture Notes in Mathematics, Vol. 1895, Springer, Berlin Heidelberg, 2007.

15. L. Molnár, Maps preserving the geometric mean of positive operators, Proc. Amer. Math. Soc. 137 (2009), 1763-1770.

16. L. Molnár, Maps preserving the harmonic mean or the parallel sum of positive operators, Linear Algebra Appl. 430 (2009), 3058-3065.

17. L. Molnár, Maps preserving general means of positive operators, Electron. J. Linear Algebra 22 (2011), 864-874.

18. L. Molnár, General Mazur-Ulam type theorems and some applications, to appear in a volume of the series "Operator Theory: Advances and Applications".

19. G. Nagy, Preservers for the p-norm of linear combinations of positive operators, Abstr. Appl. Anal., Volume 2014, Article ID 434121, 9 pages.

20. N.V. Rao, T.V. Tonev and E.T. Toneva, Uniform algebra isomorphisms and peripheral spectra, In: Topological Algebras (Eds. A. Malios and M. Haralampidou), Contemporary Math. 427 (2007), 401-416.

21. P. Šemrl, Comparability preserving maps on bounded observables, Integral Equations Operator Theory 62 (2008), 441-454.

22. P. Šemrl, Symmetries on bounded observables: a unified approach based on adjacency preserving maps, Integral Equations Operator Theory 72 (2012), 7-66.

23. T. Tonev, Spectral conditions for almost composition operators between algebras of functions, Proc. Amer. Math. Soc. 142 (2014), 2721-2732.

24. T. Tonev and R. Yates, Norm-linear and norm-additive operators between uniform algebras, J. Math. Anal. Appl. 357 (2009), 45-53.

\section{LAJOS MOLNÁR AND PATRÍCIA SZOKOL}

MTA-DE "Lendület" Functional Analysis Research Group, Institute of Mathematics University of Debrecen, H-4010 Debrecen, P.O. Box 12, Hungary

e-mail: molnarl@science.unideb.hu

URL: http://www. math.unideb.hu/ molnarl/

e-mail: szokolp@science.unideb.hu 